\title{
Computational Model of a Novel, Two-Cup Horizontal Wind-Turbine System
}

\author{
Greg Mowry, Robert Erickson and John Abraham** \\ School of Engineering, University of St. Thomas, St. Paul, MN 55105-1079, USA
}

\begin{abstract}
Typical wind turbine systems are sufficiently large so as to require extensive physical space for their installation and operation. These requirements preclude the use of turbines in crowded, urban environments. On the other hand, smaller turbine systems may find practical application as rooftop units, installed atop tall buildings. Such rooftop units must be much smaller than their ground-based counter parts. In this paper, a new, vertical-axis wind turbine has been analyzed by using a two-step numerical procedure. The design consists of two turbine cups that are positioned with $180^{\circ}$ separation. In the first step of the analysis, a complete numerical simulation of the wind-flow patterns across the cup with wind impacting angles spanning $360^{\circ}$ was completed. From these calculations, it was possible to determine the functional relationship between rotational forces, relative wind speed, and the relative angle of wind approach. The second stage of numerical procedure was a time-wise integration of the instantaneous angular velocity of the wind turbine. These calculations were carried out until the turbine had achieved quasi-steady motion. The corresponding cycle-averaged angular velocity (terminal angular velocity) was then determined. This second stage was completed for a wide range of wind speeds so that a functional dependence of the turbine rotational velocity on the wind speed could be found. This functional relationship enables a user to predict the operational response of the wind turbine based on a known and steady wind velocity.
\end{abstract}

\section{INTRODUCTION}

Wind-based electrical generation is a fast-growing source of clean power production [1]. Typically, wind-based electricity is produced in large, relatively remote wind-farms

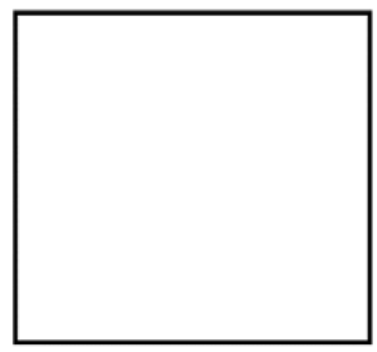

Front View

Fig. (1). Front and side view of cup design.

which are constituted by turbine populations that vary from tens to hundreds of units. Electricity is then transferred to residential and commercial centers through extensive power grids.

Another future mode of wind-energy generation is through on-sight production and utilization. In this mode,

*Address correspondence to these authors at the School of Engineering, University of St. Thomas, St. Paul, MN 55105-1079, USA; Tel: 651-962-

5766; E-mail: jpabraham@stthomas.edu wind-based electricity is generated at a residential or commercial location for immediate use. Such local wind turbines are much smaller than their wind-farm counterparts that typically reach heights of hundreds of feet above ground.

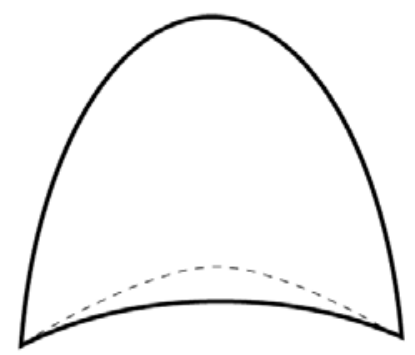

Top View

Effective local power production requires small wind turbines that are readily adapted to a variety of geometric constraints.

For grid-size wind turbines, larger and taller turbines generally perform better than smaller counterparts due to the physics of wind-power extraction and wind speeds that increase with elevation [2]. The rotational rate of grid-size wind turbines is also restricted for environmental and safety concerns. In rooftop applications, there are fewer restrictions on rotation rate and furthermore, some of the designs are even screened for safety reasons. As a consequence, the de- 

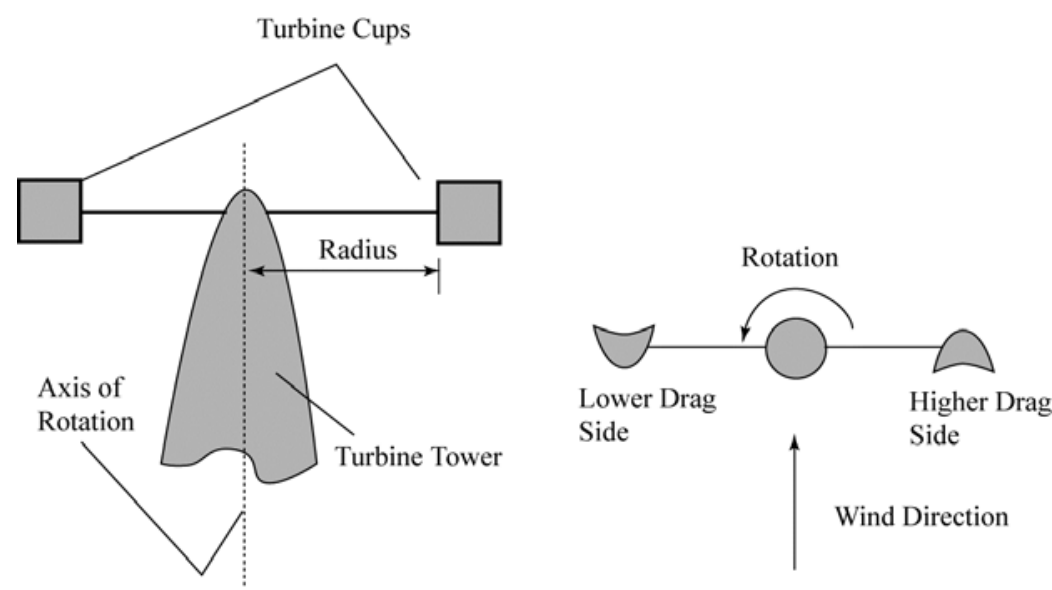

Fig. (2). Schematic diagram of the turbine system with front and side views shown.

sign space for rooftop wind turbines is quite different from their grid-sized cousins. The relatively smaller size of rooftop units also limits the power generation to a few kilowatts. Hence, for simplicity and cost reasons the associated electrical generators are typically permanent magnet machines with very efficient conversion and grid-synchronization power electronics.

In this research effort, a novel turbine system has been designed with a number of features that facilitates its use in urban or constrained environments. The major innovations are related to the use of a rectangular profiled cup design and a vertical axis of rotation. A detailed investigation of the first of these features is facilitated by reference to Fig. (1). The figure shows two views of the turbine cup. In the left view, the square profile of the front surface is shown. On the right, a top view of the cup is displayed which clearly exhibits the streamlined nature of the back surface of the cup.

When positioned in an airflow, the front and back surfaces of the cup generate differing drag forces due to their blunt and streamlined shapes. As a consequence, the turbine will rotate as shown in the rightmost portion of Fig. (2). The left half of the figure shows the turbine cups attached to a schematic tower with the vertical axis of rotation clearly indicated.

The design that is analyzed in this paper consists of two cups, located at 180 degree increments about the axis of rotation. The analysis will include a detailed numerical study of the flow of air across the cups at all circumferential locations. The outcome of the numerical study will be used in a dynamic analysis of the rotational motion. The results of the two-part study will enable a determination of the resulting rotational velocity of the new turbine design. While the analysis completed here will be specific to a specific cup design, the method is universal and would allow calculations for design variations. The profiles of the concave and convex surfaces of the blade structure are, at this point, proprietary so only the general shape of a cup will be disclosed.

\section{NUMERICAL MODEL}

\section{Fluid Flow Modeling}

The fluid modeling was completed using a finite volume computational scheme. The calculations were completed for all combinations of incoming wind speeds and directions. The complexity of the numerical simulations was great due to the rotational motion of the turbine cup which causes a continuously changing relative wind velocity and direction, even though the wind itself was assumed to be steady with regard to speed and direction. Discussion of these issues is aided by reference to Fig. (3) which shows the wind impacting at angle $\theta$ on the concave surface of the cup. As the cup completes a cycle about the axis of rotation, the angle $\theta$ varies continuously over a 360 degree range. In addition, the rotational velocity dramatically affects the incoming wind velocity relative to the cup. When the cup moves in the same direction as the wind, the relative wind velocity is decreased. On the other hand, when the motion of the cup is into the wind, the relative velocity increases.

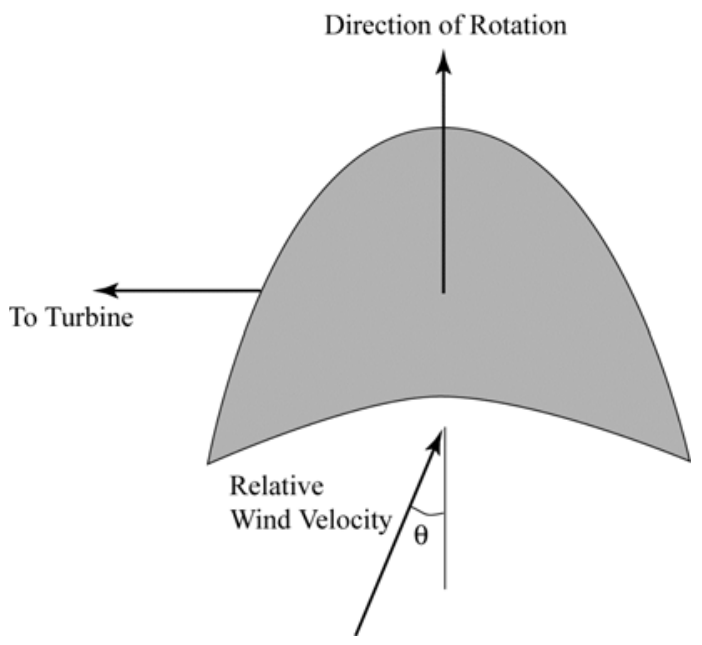

Fig. (3). Diagram showing cup and incoming wind orientation. The angle $\theta$ varies continuously over a 360 degree range.

All calculations were completed by solving equations which balance mass and momentum flowrates over all finitevolume cells which constitute the fluid region. Those equations, hereby referred to as continuity and momentum conservation, are shown in Equations (1) and (2) in tensor form [3].

$$
\frac{\partial u_{i}}{\partial x_{i}}=0
$$


$\rho\left(u_{i} \frac{\partial u_{j}}{\partial x_{i}}\right)=-\frac{\partial p}{\partial x_{i}}+\frac{\partial}{\partial x_{i}}\left(\left(\mu+\mu_{t}\right) \frac{\partial u_{j}}{\partial x_{i}}\right) \quad j=1,2,3$

All air properties are evaluated at atmospheric pressure and a temperature of $20^{\circ} \mathrm{C}$. The high velocities and the inevitable recirculation patterns guarantee that turbulence will exist within the domain. The present calculations have accommodated turbulence by means of the eddy viscosity, $\mu_{t}$, which is displayed on the right-hand side of Equation (2). The shear stress transport model (SST) of Mentor [4] has been used. That model combines the $\kappa-\varepsilon$ model of Jones and Launder [5] with the $\kappa-\omega$ approach set forth by Wilcox [6, 7]. The combination of these approaches is performed in such a manner that the $k-\omega$ equations dominate in the nearwall region while $k-\varepsilon$ holds away from the wall. In this way, the advantage of the near-wall calculations of $k-\omega$ are realized yet its sensitivity to free-stream values of the turbulent frequency is mitigated [8]. It has been shown that the SST approach provides superior results for near-wall and separated flow calculations [9-15].

The expression of the SST is provided in two extra transport equations for the turbulence kinetic energy, $k$ and the specific rate of turbulence dissipation, $\omega$. The new transport equations, are provided in Equations (3) and (4).

$\frac{\partial\left(\rho u_{i} k\right)}{\partial x_{i}}=P_{k}-\beta_{1} \rho k \omega+\frac{\partial}{\partial x_{i}}\left[\left(\mu+\frac{\mu_{t}}{\sigma_{k}}\right) \frac{\partial k}{\partial x_{i}}\right]$

and

$$
\begin{aligned}
& \frac{\partial\left(\rho u_{i} \omega\right)}{\partial x_{i}}=A \rho S^{2}-\beta_{2} \rho \omega^{2}+\frac{\partial}{\partial x_{i}}\left[\left(\mu+\frac{\mu_{t}}{\sigma_{\omega}}\right) \frac{\partial \omega}{\partial x_{i}}\right] \\
& +2\left(1-F_{1}^{\prime}\right) \rho \frac{1}{\sigma_{\omega 2} \omega} \frac{\partial k}{\partial x_{i}} \frac{\partial \omega}{\partial x_{i}}
\end{aligned}
$$

The solution of Equations (3) and (4) yields the turbulent viscosity, $\mu_{t}$, in terms of $k$ and $\omega$. It is,

$$
\mu_{t}=\frac{a \rho k}{\max \left(a \omega, S F_{2}^{\prime}\right)}
$$

where $F_{2}{ }^{\prime}$ is a blending function that limits the eddy viscosity within the boundary layer.

In these equations, $P_{k}$ is the rate of production of the turbulent kinetic energy and the $\sigma$ terms are the Prandtl numbers for transport of turbulent kinetic energy, and specific rates of turbulence destruction $(\omega$ and $\omega 2) . F_{1}$ is a blending function that facilitates the combination of the standard $\kappa-\varepsilon$ model and the $\kappa-\omega$ model. The term $S$ is the absolute value of the shear strain rate, and the $\beta$ terms are model constants.

At all solid-fluid interfaces, the no-slip condition was employed so that the fluid velocity was zero. The turbulent kinetic energy is also zero at these surfaces. A portion of the computational domain nearest the cup is shown in Fig. (4). That figure is a two-dimensional top view of the three dimensional extent of the volume under consideration. The figure, which is not drawn to scale, shows that the fluid region completely encloses the cup. Airflow is applied at two orthogonal surfaces of the fluid region. The modeled inlet condition was steady so that the direction and magnitude of the wind did not change in time (no gusts). Also, it was assumed that the wind passed parallel to the base of the wind turbine and did not spatially vary at the blade surface. The calculations were carried out for values of $\theta$ which spanned the entire $360^{\circ}$ range. They also covered the expected range of relative wind velocities.

Opening conditions are used to complete the enclosure which allows air to flow either into or out of the domain. The use of an opening condition is required by the potential for the formation of eddies downstream of the cup. A detailed

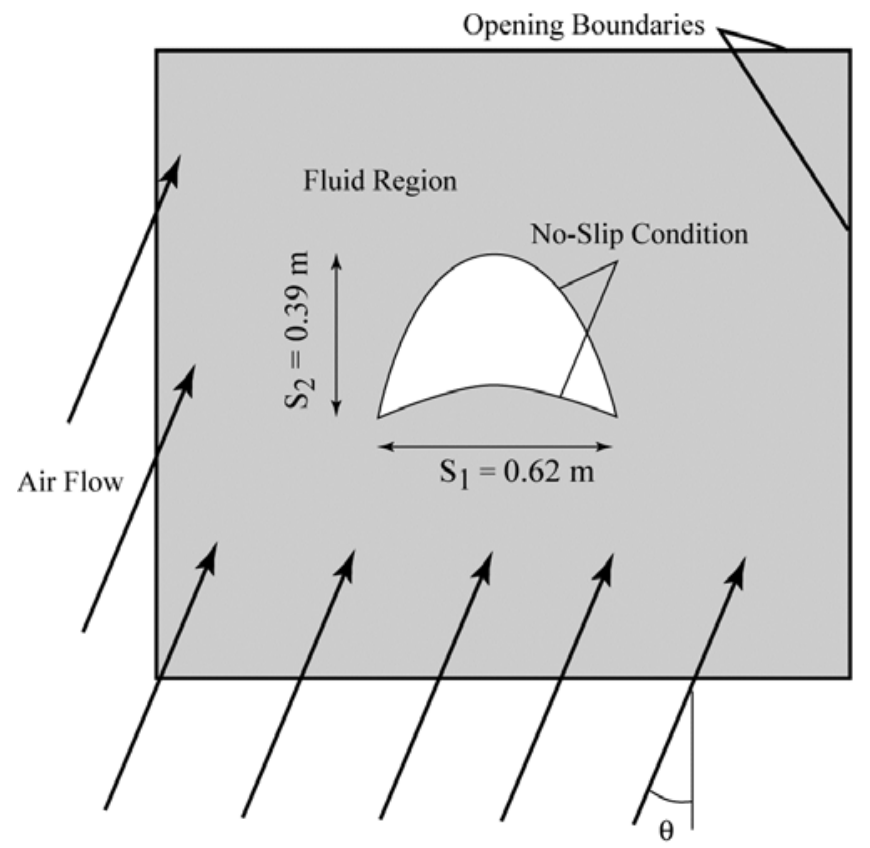

Fig (4). Boundary conditions used in the fluid simulation. 
study was made on the required extent of the solution domain so that the positioning of all boundaries were sufficiently far from the cup so as to not affect the calculations.

The fluid region was subdivided into 2,000,000 elements which were preferentially deployed in regions of high gradients, such as at all fluid-solid interfaces. To accomplish this deployment, thin prismatic elements were placed along all boundaries. A view of the element deployment is shown in Fig. (5), which is a projection of the control volumes taken from a horizontal slice through the three-dimensional region. The CFX 11.0 solver was used to complete the calculations.

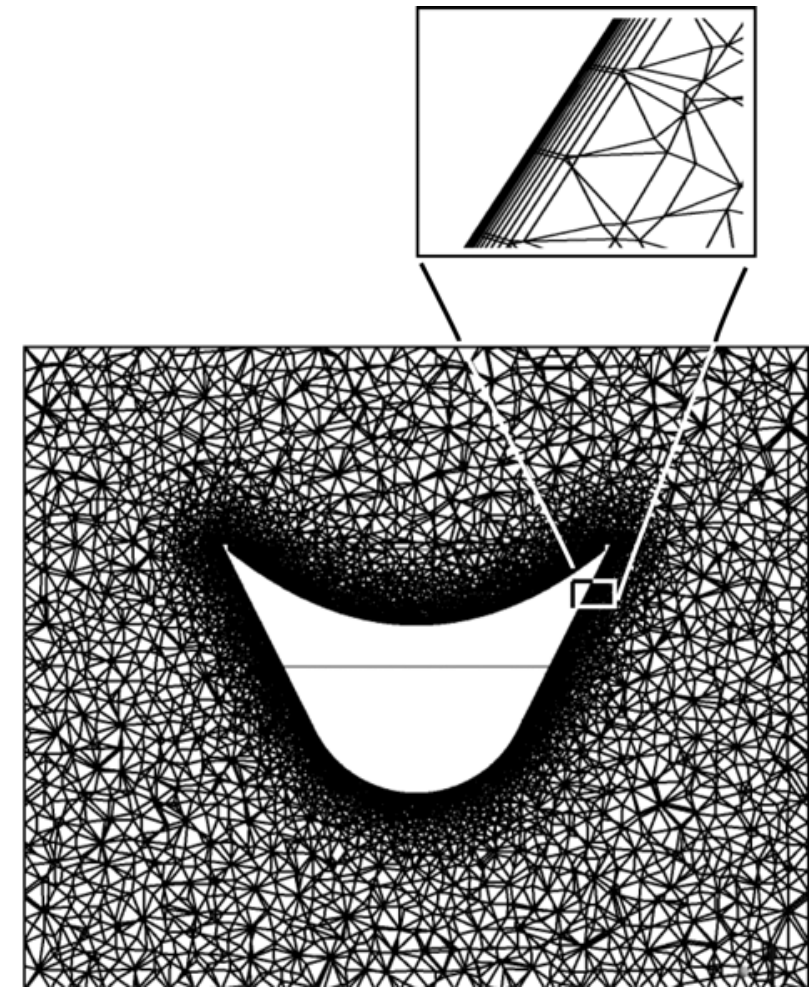

Fig. (5). Depiction of the mesh near the surfaces of the cup.
Coupling of the velocity-pressure equations was achieved on a non-staggered, collocated grid using the techniques developed in [16] and [17]. The inclusion of pressuresmoothing terms in the mass conservation equation suppresses oscillations which can occur when both the velocity and pressure are evaluated at coincident locations.

The advection terms in the momentum equations were evaluated by using the upwind values of the momentum flux, supplemented with an advection-correction term. The correction term reduces the occurrence of numerical diffusion and is of second-order accuracy. Further details of the advection treatment can be found in [18].

The calculations were performed using a false-transient algorithm. Mesh and time-step values were sufficiently small to ensure a solution that was independent of their values. The selected values resulted from an independence study during which both the element sizes and time steps were reduced and results were compared. When the sequential reductions failed to yield noticeable changes in the results, it was determined that the settings were sufficiently refined.

\section{Model of Rotational Motion}

The calculations of the proceeding section were completed for multiple angles $\theta$ and for a number of relative wind velocities. For each calculation, the circumferential force was determined by integrating pressure and shear forces over all surfaces of the cup. The resulting circumferential force, which at all instances is tangent to the direction of motion, provides the torque about the axis of rotation. A diagram of the circumferential forces on the two cups is shown in Fig. (6). It is worthy of note that in most positions, the two forces provide opposing moments, with one force acting to continue rotation and the other acting to slow the rotational velocity.

With the two forces now determined, it is possible to construct an equation which describes the rotational motion of the two-cup system. That equation, shown below contains

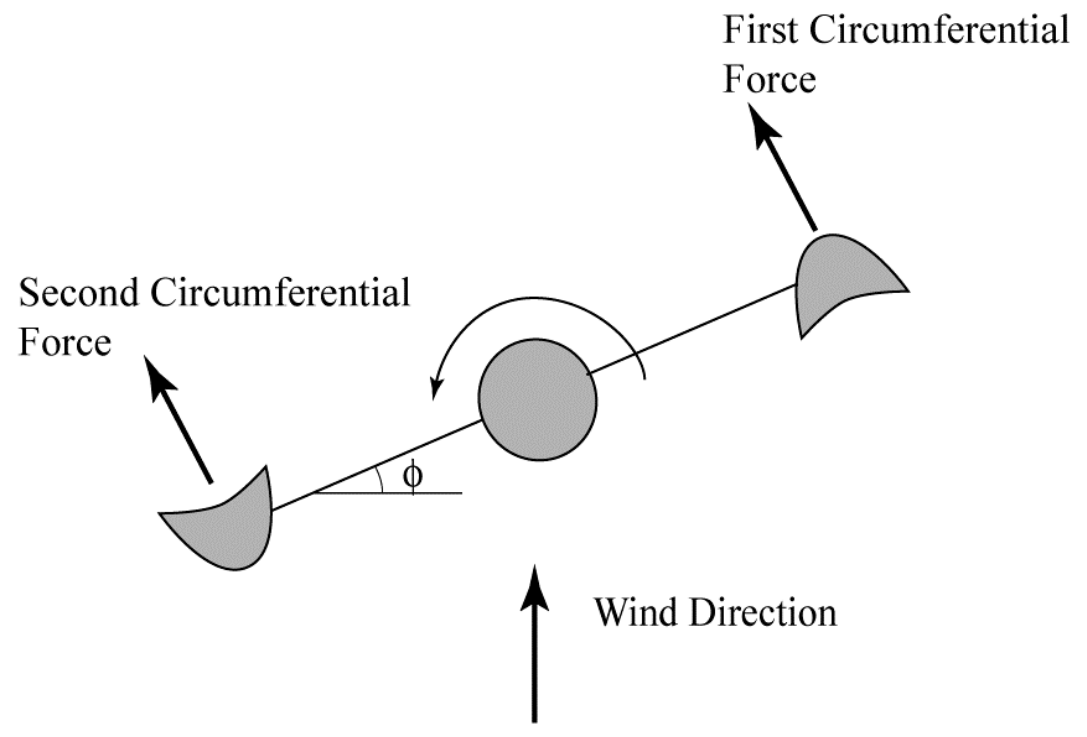

Fig. (6). Schematic showing circumferential forces acting on turbine cups 
the moment of inertia of the system, $I=2.9 \mathrm{~kg} \mathrm{~m}^{2}$, and the instantaneous angular acceleration, $\alpha$.

$$
\text { I } \alpha=M_{1}-M_{2}=\left(F_{1}-F_{2}\right) \cdot \text { Radius }
$$

The model, as presented in Equation 6 ignores frictional losses due to rotation, and drag forces in the thin beams which connect the cups to the turbine.

In Equation (6), $F_{1}$ and $F_{2}$ are, respectively, the forces promoting and opposing rotation. As evident from the description of the fluid flow calculations, the two forces $F_{l}$ and $F_{2}$ are continuous functions of both the relative wind speed and of the angle of incidence of the wind. The functional dependence of the circumferential forces can be represented as,

$$
F=f\left(\theta,\left|V_{r e l}\right|\right)
$$

When this expression is inserted into Equation (6), the motion equation becomes,

$$
I \frac{d \omega^{\prime}}{d t}=\left(f\left(\theta_{1},\left|V_{\text {rel }}\right|_{1}\right)-f\left(\theta_{2},\left.\left|V_{\text {rel }}\right|\right|_{2}\right)\right) \cdot \text { Radius }
$$

In Equation (8), $\omega^{\prime}$ is the instantaneous angular velocity and is equal to,

$$
\frac{d \varphi}{d t}=\omega^{\prime}
$$

where angle $\phi$ is the angle of inclination of the turbine system with respect to the wind, as shown in Fig. (6).

Equations (8) and (9) completely determine the progression of motion. The non-linearity of the system requires that the solution of these coupled equations be obtained numerically using a time-stepping solution procedure. The algorithm provides the progression of the angular position and velocity of the turbine based on initial conditions for both $\phi$ and $\omega^{\prime}$.

Using the symbol $n$ to reference the current time step, Equation (8) is evaluated based at the current time-step as shown in Equation (10).

$$
I \frac{d\left(\omega^{\prime}\right)^{n}}{d t}=\left(f\left(\theta_{1},\left|V_{\text {rel }}\right|_{1}\right)^{n}-f\left(\theta_{2},\left|V_{\text {rel }}\right|_{2}\right)^{n}\right) \cdot \text { Radius }
$$

Then, with $\left(\omega^{\prime}\right)^{n}$ determined, the incremental change in the angular position and velocity of the turbine is calculated from a forward-stepping integration, as shown in the following:

$\varphi^{n+1}=\left(\omega^{\prime}\right)^{n} \cdot \Delta t+\varphi^{n}$

and

$$
\left(\omega^{\prime}\right)^{n+1}=(\alpha)^{n} \cdot \Delta t+\left(\omega^{\prime}\right)^{n}
$$

where $\alpha$ is the angular acceleration. In all calculations, the time step was selected to ensure both numerical stability and accuracy. Accuracy was ensured by successively reducing the numerical integration time steps until no difference in outcome was observed.

The new information is used to update the wind speed and angle of incidence which then allow a determination of newly updated forces $F_{1}$ and $F_{2}$, and a continuation of the calculation procedure.

The time-stepping calculations of Equations (11) and (12) were carried out until the wind turbine reached a quasisteady motion. The motion of the turbine accelerated during part of its rotation and decelerated during other portions. Quasi-steady motion is achieved when the time integrated acceleration equaled the same integration of deceleration throughout one complete cycle. For quasi-steady motion, the instantaneous angular velocities evaluated at one cycle are identical to those evaluated at a subsequent cycle.

\section{RESULTS AND DISCUSSION}

\section{Fluid Flow Results}

Results from the fluid analysis are best illustrated by visualization of the flow field. To facilitate the following

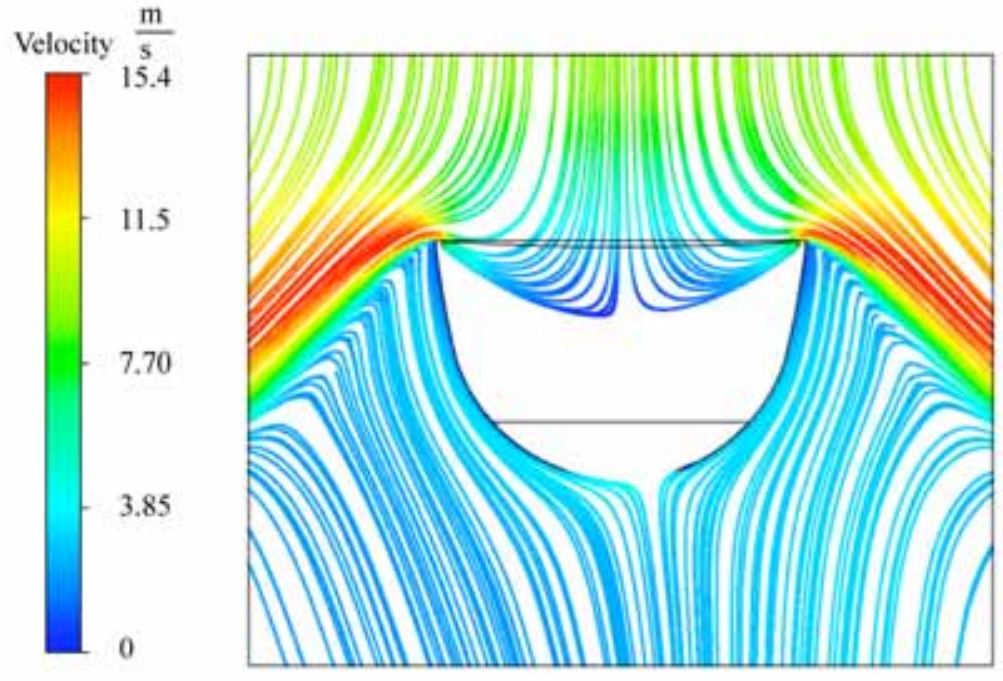

Fig. (7). Streamline pattern for flow with an incident angle of 0 degrees, color-coded by velocity magnitude corresponding to a relative wind speed of 20 miles/hour $(9 \mathrm{~m} / \mathrm{s})$. 

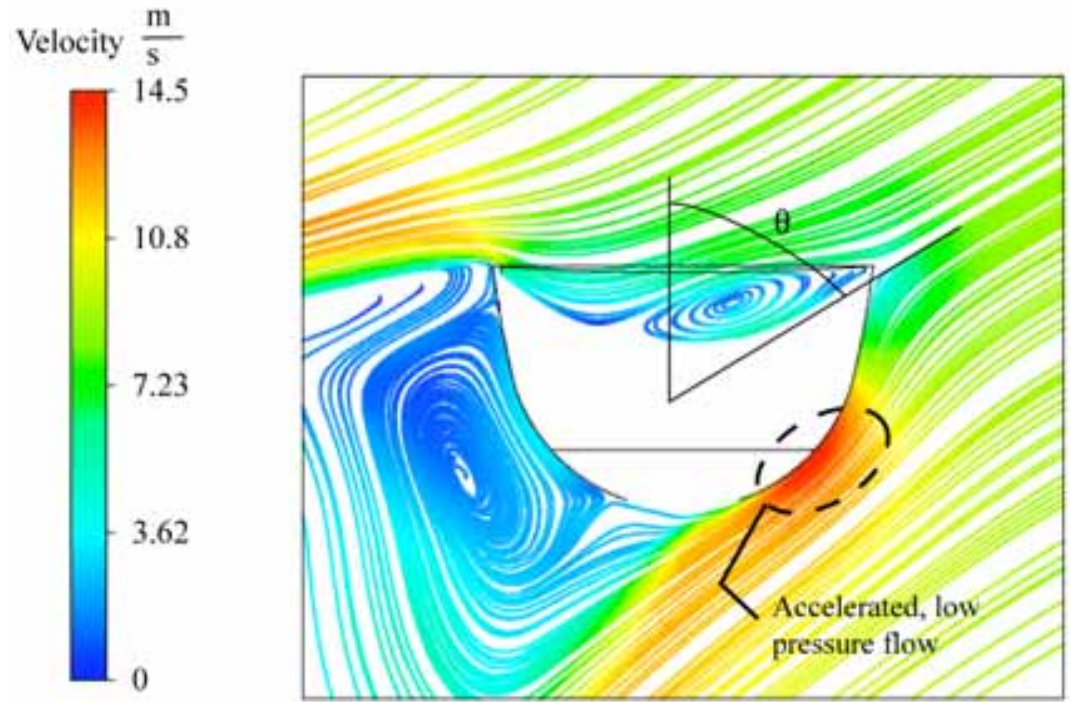

Fig. (8). Streamline pattern for flow with an incident angle of 60 degrees, color-coded by velocity magnitude corresponding to a relative wind speed of 20 miles/hour $(9 \mathrm{~m} / \mathrm{s})$.

discussion, representative results corresponding to airflow angles-of-incidence of 0, 60 and 120 degrees are shown. The first set of results is displayed in Fig. (7), which shows streamlines of the flow passing normal to the concave surface of the cup. The streamlines, which are color-coded by the velocity scale on the left side of the figure, have been obtained on a two-dimensional cross-sectional cut through the cup and fluid region. It should be noted that the solution was, in fact, fully three dimensional. The results of Fig. (7) are for an incident velocity of 20 miles per hour $(9 \mathrm{~m} / \mathrm{s})$. The deflection of streamlines on the front face of the cup is evident, as is the separation of flow on the downstream surfaces. In the figure, the airflow passes downwards, over the front surface of the cup.
The aforementioned deflection of flow at the front surface of the cup causes a rise in local air pressure as the kinetic energy of the air is converted to pressure. The pressure and shear stress distributions across the entire surface of the cup were integrated to provide the overall net circumferential force which results in rotation of the turbine blade.

A corollary set of figures for the cases of incident angles of 60 and 120 degrees are presented in Figs. (8) and (9), respectively. The figures show the streamline pattern including separation and the pressure distribution on the front face of the cup. Of note is a region of high velocity flow which, with its corresponding low pressure, creates a component of tangential force (cup lift) which aides in rotating the turbine blade.

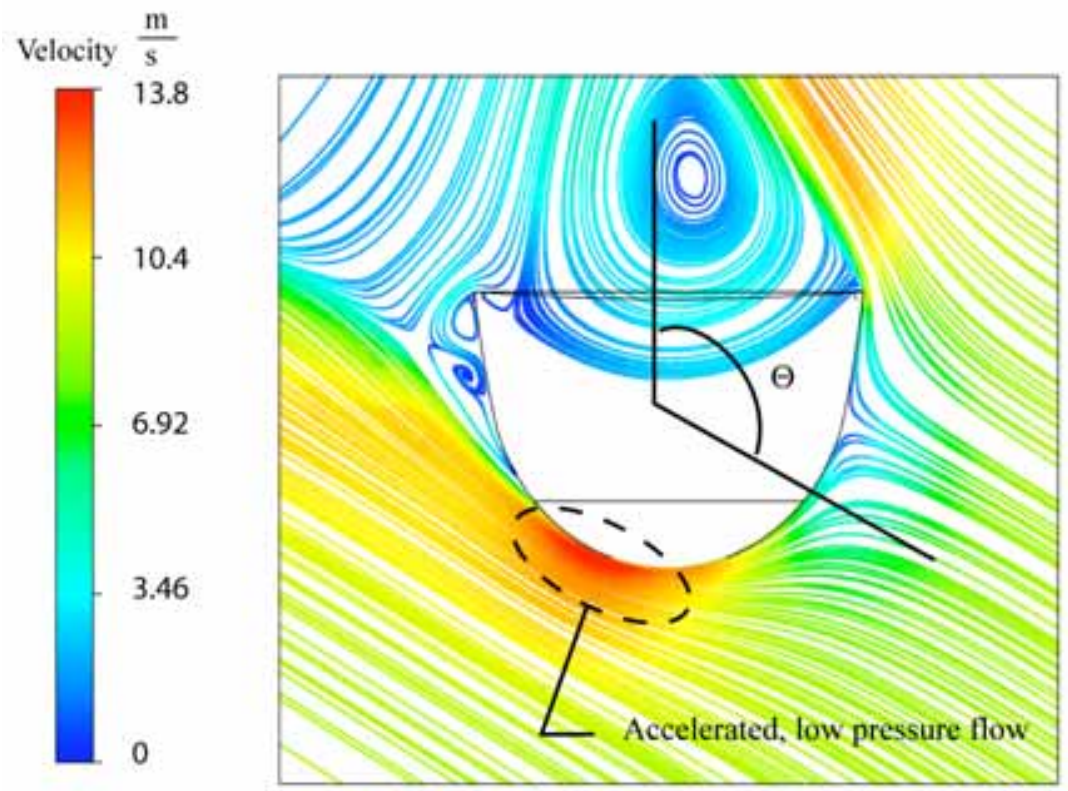

Fig. (9). Streamline pattern for flow with an incident angle of 120 degrees, color-coded by velocity magnitude corresponding to a relative wind speed of 20 miles/hour $(9 \mathrm{~m} / \mathrm{s})$. 


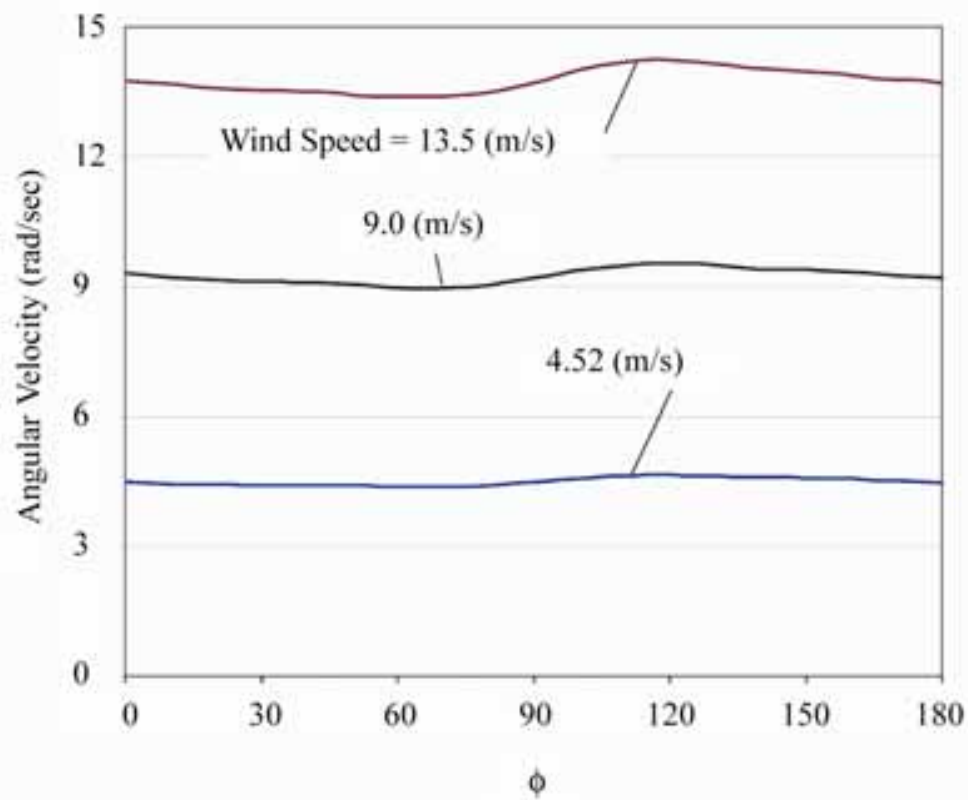

Fig. (10). Values of the angular velocity for wind speeds of $4.52,9.0$, and $13.5(\mathrm{~m} / \mathrm{s})$ which correspond, respectively, to 10,20 , and 30 miles/hour.

The flow depictions set forth in Figs. (7-9) are illustrative and serve to demonstrate the complicated flow patterns which exist in the region near the turbine cup. Similar results have been obtained for multiple angles $\theta$ and relative wind velocities but are not shown here for brevity.

\section{Rotational Motion Results}

With the flow field calculations completed and tangential forces available at multiple relative wind velocities and angles, the numerical integration shown in Equations (11) and (12) can be completed. The calculations utilized a time-step increment, $\Delta t$, of 0.01 seconds. The calculations were continued from an imposed initial value of both $\omega^{\prime}$ and $\phi$ until a quasi-steady state was reached. The achievement of quasisteady motion was determined when the cycle-to-cycle variation of the angular velocity $\omega^{\prime}$ was less than $1 \%$.
The cycle-average angular velocity which corresponds to the quasi-steady state, hereafter called the terminal angular velocity, was obtained for multiple wind velocities so that the turbine response can be determined for any wind speed. A depiction of sample results is shown in Fig. (10) where the angular velocity is shown for an entire cycle and for three wind velocities, $4.52,9.0$, and $13.5 \mathrm{~m} / \mathrm{sec}$, which are equivalent to, respectively 10,20 , and 30 miles per hour. The figure shows results for a $180^{\circ}$ variation of the angle $\phi$. The two cup system experiences periodic motion so that the pattern of angular velocity is repeated for every $180^{\circ}$ of motion.

For all cases presented in Fig. (10), it is seen that the angular velocity of the turbine varies slightly throughout the cycle. The terminal angular velocity for a given case is calculated by integrating the instantaneous angular velocity over the entire period of motion. When the terminal velocity

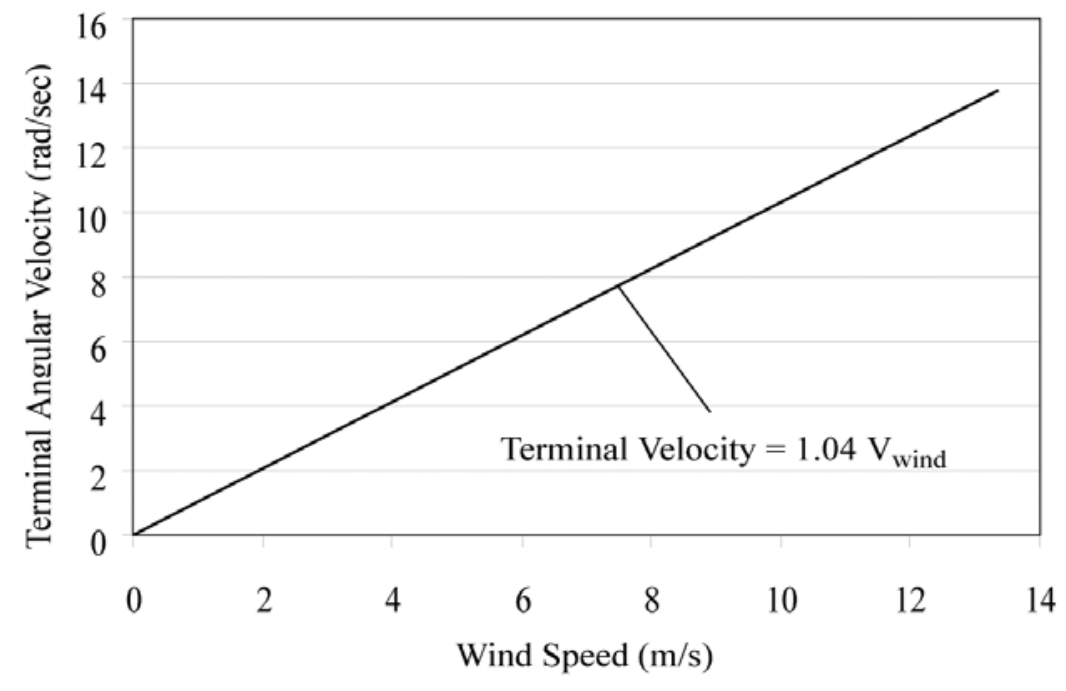

Fig. (11). Variation of terminal velocity with wind speed. 
is obtained for a sequence of wind velocities, it is possible to develop a functional relationship between the two variables. Such a functional relationship is shown graphically in Fig. (11).

The results of Fig. (11) show that the terminal velocity depends linearly on the magnitude of the wind speed. This linear dependence is somewhat unexpected because in general, the circumferential force exerted on the cup varies as $\mathrm{V}_{\text {wind }}{ }^{2}$. On the other hand, it must be recognized that for most of the rotational period, the two cups experience two, counteracting forces. This fact tends to diminish the sensitivity of the angular velocity on the wind speed. The data illustrated in Fig. (11) has great utility in that it can be used to predict the final rotational velocity of the wind turbine for any incoming, steady wind speed and ultimately, the power generated by the wind turbine.

Of critical importance is the expected power generation which results from the rotating turbine. Based on the rotational results obtained with this candidate two-cup turbine design, the electrical output will be 12,100 , and 325 watts, respectively for wind speeds of $4.5,9$, and 13.5 meters per second.

\section{CONCLUDING REMARKS}

A two-step numerical simulation has been used to evaluate the efficacy of a new, vertical axis, small-scale wind turbine. The turbine possesses cups with a square front face and a smoothly contoured rear body which results in a net positive moment when the turbine is positioned in blowing air. Other features of the turbine include its very small profile which facilities its use in crowded, urban rooftop applications.

The numerical analysis consisted of a detailed simulation of the airflow patterns which exist across the cup surfaces. Calculations were made for a wide range of approach velocities and angles. These results enabled the continuous calculations of circumferential forces to be made on a two-cup system. Based on the circumferential forces, it was possible to determine the quasi-steady rotational motion of the turbine for a collection of steady wind velocities. The calculations ignored frictional losses within the turbine housing. Calculations of the rotary motion were completed using a forwardstepping numerical integration in time. Convergence was determined when the cycle-to-cycle variation in the angular velocity was less than $1 \%$.

The results presented here are summarized in a function which relates the terminal angular velocity to the wind speed. That functional relationship enables a user to predict angular velocity of the turbine system.

\section{ACKNOWLEDGEMENTS}

The authors would like to acknowledge Horizontal Winds for the grant to the University of St. Thomas' School of Engineering. This grant enabled the directed student research that was instrumental to the success of the project.

\section{NOMENCLATURE}

$a$

Turbulent model constant
A

$F_{1}$ and $F_{2} \quad$ Circumferential forces [N]

$F_{1}{ }^{\prime}$ and $F_{2}{ }^{\prime} \quad$ Turbulent model blending functions

i,j Tensor indices

I $\quad$ Moment of inertia $\left[\mathrm{kg} \mathrm{m}^{2}\right]$

$k$

$M_{1}$ and $M_{2} \quad$ Moments

$p \quad$ Pressure $[\mathrm{Pa}]$

$P_{k} \quad$ Turbulence model production term

$S \quad$ Shear strain rate

$t \quad$ Time [s]

$u_{i} \quad$ Local velocity $[\mathrm{m} / \mathrm{s}]$

$V_{\text {rel }} \quad$ Relative wind velocity $[\mathrm{m} / \mathrm{s}]$

$x \quad$ Coordinate direction [m]

Greek

$\begin{array}{ll}\alpha & \text { Angular acceleration of turbine }\left[\mathrm{rad} / \mathrm{s}^{2}\right] \\ \beta_{l} \text { and } \beta_{2} & \text { Turbulent model constants } \\ \theta & \text { Incident angle of wind } \\ \phi & \text { Angular position of turbine } \\ \omega & \text { Specific rate of turbulence dissipation } \\ \omega^{\prime} & \text { Angular velocity [rad } / \mathrm{s}] \\ \rho & \left.\text { Air density [kg/m } \mathrm{m}^{3}\right] \\ \mu & \text { Molecular viscosity }\left[\mathrm{N} \mathrm{s} / \mathrm{m}^{2}\right] \\ \mu_{t} & \left.\text { Turbulent viscosity [N s/m } \mathrm{m}^{2}\right] \\ \sigma & \text { Prandtl number }\end{array}$

\section{REFERENCES}

[1] "Annual report on U.S. wind power installation, cost, and performance trends: 2007", U.S. Department of Energy, May 2008.

[2] T. Ackermann, Ed., Wind Power in Power Systems. New York: John Wiley and Sons, 2005.

[3] W. Kays, M. Crawford, and W. Bernhard, Convective Heat and Mass Transfer, $4^{\text {th }}$ ed. London: McGraw-Hill, 2004.

[4] F. Menter, "Two-equation eddy-viscosity turbulence models for engineering applications", AIAA J., vol. 32, pp.1598-1605, 1994.

[5] B. Launder and D. Spalding, "Numerical computation of turbulent flows”, Comput. Meth. Appl. Mech. Eng., vol. 3, pp. 269-289, 1974. 
[6] D. Wilcox, "Re-assessment of the scale-determining equation for advanced turbulence models", AIAA J., vol. 26, pp. 1299-1310, 1988.

[7] D. Wilcox, "Comparison of two-equation turbulence models for boundary layers with pressure gradient", AIAA J., vol. 32, pp. 1414-1421, 1993.

[8] F. Menter, "Influence of freestream values on k-omega turbulence model predictions", AIAA J., vol. 30, pp. 1657-1659, 1992.

[9] M. Malone, "Turbulence model evaluation for free shear dominated flows", AIAA paper 1996-2038, New Orleans, LA, 1996.

[10] J. Ladd and T. Korakianitis, "On the assessment of one- and twoequation turbulence models for the computation of impinging jet flowfields", AIAA paper 1996-2545, 1996.

[11] R. Bush, "A two-equation large eddy stress model for high sub-grid shear", AIAA Paper 2001-2561, 2001

[12] C. Rumsey, T. Gatski, and J. Morrison, "Turbulence model predictions of strongly curved flow in a U-duct", AIAA J., vol. 38, pp. $1394-1402,2000$
[13] J. Ekaterinas, "Computation of oscillating airfoil flows with oneand two-equation turbulence models", AIAA J., vol. 32, pp. 23592365, 1994.

[14] F. Menter, M. Kuntz, and R. Langtry, "Ten years of industrial experience with the SST turbulence model", in International Symposium on Turbulence Heat and Mass Transfer 4, Antalya, Turkey, October 12-17, 2003.

[15] J. Abraham and A. Thomas, "Induced co-flow and laminar-toturbulent transition with synthetic jets", Comput. Fluids, (in press).

[16] C. Rhie and W. Chow, "A numerical study of the turbulent flow past an isolated airfoil with trailing edge separation", AIAA paper 82-0998, 1982.

[17] S. Majumdar, "Role of underrelaxation in momentum interpolation for calculation of flow with nonstaggered grids", Num. Heat Transfer, vol. 13, pp. 125-132, 1998.

[18] T. Barth and D. Jesperson, "The design and applications of upwind schemes on unstructured meshes", AIAA paper 89-0366, 1989.

Received: November 18, 2008

Revised: February 16, 2009

Accepted: February 18, 2009

(C) Mowry et al.; Licensee Bentham Open.

This is an open access article licensed under the terms of the Creative Commons Attribution Non-Commercial License (http://creativecommons.org/licenses/bync/3.0/), which permits unrestricted, non-commercial use, distribution and reproduction in any medium, provided the work is properly cited. 